Original Article

\title{
DESIGN AND DEVELOPMENT OF RILPIVIRINE NANOPARTICLE CONTAINING CHITOSAN USING IONIC GELATION METHOD FOR HIV INFECTIONS
}

\author{
MONTUKUMAR PATEL ${ }^{1 *}$, NIRAV V. PATEL1, TEJAS B. PATEL ${ }^{2}$ \\ ${ }^{*}$ 1Department of Pharmaceutical Science, Saurashtra University, Rajkot, Gujarat, India, ${ }^{2}$ Faculty of Pharmacy, Dharmsinh Desai University, \\ Nadiad, Gujarat, India \\ Email: montu9689@gmail.com
}

Received: 21 Sep 2019, Revised and Accepted: 21 Dec 2019

\begin{abstract}
Objective: The primary objective of the current research was to prepare rilpivirine loaded Nanoparticles containing Chitosan using the ionic gelation method for HIV infections.

Methods: The nanoparticles of rilpivirine were prepared using the ionic gelation technique. Further, nanoparticles were characterized by Fourier transform infrared spectroscopy (FTIR), differential scanning calorimetry (DSC), scanning electron microscopy (SEM) and in vitro drug release.

Results: The optimized nanoparticles were found with a particle size of $130.30 \pm 5.29 \mathrm{~nm}$ (mean $\pm \mathrm{SD}$ ) and entrapment efficiency (\% EE) of $77.10 \pm 0.50 \%$. Scanning electron microscopy technique exposed spherical particles with uniform size. It was observed that the nanoparticles created showed the absence of the crystalline nature of the drug and its switch to the amorphous state. Results showed that more than $45 \%$ of the pure drug is released in $50 \mathrm{~min}$ and after $90 \mathrm{~min}$ almost about $95 \%$ of the drug is released.
\end{abstract}

Conclusion: The research study concluded that the in vitro release profile of nanoparticles was found to be sustained up to $24 \mathrm{hr}$. Sustained release of the rilpivirine could improve patient obedience to drug regimens, growing action effectiveness.

Keywords: Rilpivirine, Chitosan, Nanoparticles, HIV

(c) 2020 The Authors. Published by Innovare Academic Sciences Pvt Ltd. This is an open-access article under the CC BY license (http://creativecommons.org/licenses/by/4.0/D DOI: http://dx.doi.org/10.22159/ijpps.2020v12i2.35814. Journal homepage: https://innovareacademics.in/journals/index.php/ijpps

\section{INTRODUCTION}

Chitosan is an interesting natural material occurring in abundance in the environment. Its excellent biocompatibility and several advantages due to its unique polymer cationic character render it highly useful for pharmaceutical applications. A polysaccharide comparable to cellulose, comprising copolymers of glucosamine and $\mathrm{N}$-acetyl glucosamine linked by $\beta-(1-4)$ linkages, chitosan can be derived by partial deacetylation of chitin from crustacean shells. The primary amino groups lead to special properties that render chitosan very interesting for pharmaceutical applications. In contrast to most other natural polymers, it has a positive charge and is mucoadhesive. Besides other applications, chitosan has been extensively examined for its potential in the development of controlled release drug delivery systems and these controlled release formulations have been made in the form of chitosan gels, tablets, and capsules, in addition to microspheres and microcapsules [1-3].

Nanoparticles have a special role in targeted drug delivery in the sense that they have all the advantages of liposomes including the particle size, but unlike liposomes, nanoparticles have a long shelf life and can usually entrap more drugs than liposomes. Although nanoparticles made of hydrophobic polymers encapsulating hydrophobic drugs have been reported in the literature, very few studies have been made in the preparation of drug-loaded hydrogel nanoparticles. Nanoparticles made of hydrophobic polymers are usually taken up by the Reticuloendothelial system (RES) and have a short residence time in blood. The surfaces of these hydrophobic nanoparticles are made hydrophilic by conjugating with the polyethylene glycol (PEG) type of molecules to make these particles long circulating in blood [4,5]. We have recently optimized a method of preparation of ultra-low size nanoparticles of hydrogel polymers and have been able to load water-soluble drugs into them. These hydrophilic nanoparticles evade RES and remain in circulation for a couple of hours without PEG conjugation on the particle surface [6]. These hydrogel polymers can have reactive groups on the surface which enable the nanoparticles to be converted to stimuli-responsive particles and they can also be made targetable by attaching receptor-specific ligands. The preparation of nanoparticles from chitosan, a hydrogel polymer, for drug delivery can have several advantages over the use of chitosan microspheres and microcapsules $[7,8]$.

Nanometer range particles have easy accessibility in the body, being transported via the circulation to different body sites. Extremely small nanoparticles, of $<100 \mathrm{~nm}$ diameter with a hydrophilic surface, have been found to have longer circulation in blood. Such systems should allow the control of the rate of drug administration that prolongs the duration of the therapeutic effect, as well as the targeting of the drug to specific sites [9]. The preparation of nanoparticles with this versatile hydrogel material has been attempted by several workers to utilize its mucoadhesive properties to transport drugs and DNA across a mucosal surface. The polycationic nature of deacetylated chitosan results in polycondensation in the presence of anionic macromolecules [10, 11]. Thus, Chitosan-DNA nanoparticles can be prepared by the method of coacervation. Besides, the reactive free amino group on the particle surface makes it possible to chemically conjugate various other reactive groups, such as polyethylene glycol (PEG) derivatives, different ligands, antibodies, and other $\mathrm{pH}$ and temperature-sensitive moieties. Several methodologies have also incorporated other polymers in the preparative procedure, with the intent of preparing smart hydrophilic nanoparticles under extremely mild conditions [12-14].

Considering the advantages of using ultra-low sized hydrophilic nanoparticles for drug delivery, we have focused on the preparation of chitosan nanoparticles of $<100 \mathrm{~nm}$ diameter. The preparation of nanoparticles using reverse micelles as a medium makes it possible to produce ultrafine particles with narrow size distribution. The aqueous core of the reverse micellar droplets can be used as a nanoreactor to prepare these particles since the size of the reverse micellar droplets is in the nanometer range and these droplets are highly monodispersed. The present paper describes the preparation of cross-linked chitosan nanoparticles of size $<100 \mathrm{~nm}$ diameter 
using reverse micelles as the media and their physicochemical characterization as well as biodistribution.

\section{MATERIALS AND METHODS}

\section{Materials}

Rilpivirine was obtained as a gift from Sun Pharma Advanced Research Center (SPARC), Vadodara, Guj. India. Chitosan was gifted from the Central Institute of Fisheries Technology, Cochin. All the other ingredients and solvents used in the preparation of nanoparticles were used as obtained and are of analytical grade.

\section{Preparation of rilpivirine loaded chitosan nanoparticles}

Rilpivirine loaded chitosan nanoparticles were prepared [15, 16]. with some modifications based on the ionic gelation of rilpivirine with tripolyphosphate (TPP) anions. Chitosan was dissolved in the $1 \%$ acetic aqueous solution and rilpivirine was dissolved in $2 \%$ tween-80 solutions, which was added to the chitosan solution. Under mechanical stirring (1000 rpm) (Remi, India) at room temperature, $0.25 \%$ sodium tripolyphosphate aqueous solution was added dropwise into drug and polymeric mixture for $30 \mathrm{~min}$. Thereafter, the rilpivirine loaded chitosan particle suspension was processed under ultra-sonication by probe sonicator for $10 \mathrm{~min}$, producing nanoparticles with controlled particle sizes. The dispersion was stored in a refrigerator until further evaluation. The arrangement of the nanoparticle was a result of the interface between the negative groups of the TPP and the positively charged amino groups of chitosan (ionic gelation). The formulation composition for chitosan loaded nanoparticles was depicted in table 1.

Table 1: Formulation consideration of chitosan nanoparticles

\begin{tabular}{llll}
\hline Batch code & Rilpivirine $(\mathbf{m g})$ & $\mathbf{0 . 2 5} \%$ TPP solution $(\mathbf{m l})$ & Rilpivirine $(\mathbf{m g})$ : chitosan 0.1 \% w/v (ml) \\
\hline MJ-1 & 25 & 10 & $1: 1$ \\
MJ-2 & 25 & 10 & $1: 2$ \\
MJ-3 & 25 & 10 & $1: 3$ \\
MJ-4 & 25 & 10 & $1: 4$ \\
MJ-5 & 25 & 10 & $1: 5$ \\
MJ-6 & 25 & 10 & $1: 6$ \\
\hline
\end{tabular}

\section{Preliminary investigation of formulation for nanoparticles}

Optimization of the nanoparticles was carried out using a varying amount of $1 \%$ chitosan and $0.25 \%$ TPP solution. Two different amounts of $1 \%$ chitosan i.e. 15 and $20 \mathrm{ml}$ and three different amounts of $0.25 \%$ TPP viz. 6,8 , and $10 \mathrm{ml}$ were used to screen the best batchbased in the particle size and entrapment efficiency of nanoparticles. The batches were coded from M-1 to M-6 as shown in table 2.

Table 2: Optimization parameters

\begin{tabular}{lll}
\hline Batch & Chitosan $\mathbf{0 . 1} \% \mathbf{~ w} / \mathbf{v}(\mathbf{m l})$ & $\mathbf{0 . 2 5} \% \mathbf{T P P}$ solution(ml) \\
\hline M-1 & 15 & 10 \\
M-2 & 15 & 08 \\
M-3 & 15 & 06 \\
M-4 & 20 & 10 \\
M-5 & 20 & 08 \\
M-6 & 20 & 06 \\
\hline
\end{tabular}

The rilpivirine loaded chitosan nanoparticles were prepared in six batches keeping the drug and $0.25 \%$ TPP constant at $25 \mathrm{mg}$ and $10 \mathrm{ml}$ respectively. The ratio for the drug (rilpivirine): chitosan was changed from 1:1 to 1:6 and coded as MJ-1 to MJ-6. Composition parameters are shown in table 1.

\section{Characterization of nanoparticles}

\section{Determination of entrapment efficiency (\%)}

Entrapment efficiency gives an idea about the \%drug that is successfully entrapped/adsorbed into nanoparticles. Rilpivirine in the nanoparticles was analyzed by dissolving the nanoparticles in $0.5 \%$ Polysorbate-20 in $0.01 \mathrm{~N} \mathrm{HCl}(\mathrm{pH} 1.2)$. The concentration of nanoparticles in $0.5 \%$ polysorbate- 20 in $0.01 \mathrm{~N} \mathrm{HCl}$ was $10 \mathrm{mg} / \mathrm{ml}$. The mixture was stirred continuously overnight at room temperature. Solutions were then analyzed by UV-Vis spectrophotometer at $304 \mathrm{~nm}$ with suitable dilutions [17]. The encapsulation efficiency was calculated with the help of the following formula.

$$
\% \mathrm{EE}=\frac{\mathrm{TDC}-\mathrm{D}_{\mathrm{f}}}{\mathrm{TDC}} \times 100
$$

Where, $\mathrm{TDC}=$ total drug content, $\mathrm{D}_{\mathrm{f}}=$ free drug

\section{Surface morphology}

Scanning electron microscopy (SEM) is a method for high-resolution imaging of surfaces. The SEM uses electrons for imaging, much as a light microscope uses visible light. The advantages of SEM over light microscopy include much higher magnification $(>100,000 \mathrm{X})$ and greater depth of field up to 100 times that of light microscopy. Qualitative and quantitative chemical analysis information is also obtained using an energy dispersive x-ray spectrometer (EDS) with the SEM [18]. The SEM generates a beam of incident electrons in an electron column above the sample chamber. The electrons are produced by a thermal emission source, such as a heated tungsten filament, or by a field emission cathode. The energy of the incident electrons can be as low as $100 \mathrm{eV}$ or as high as $30 \mathrm{keV}$ depending on the evaluation objectives. The electrons are focused into a small beam by a series of electromagnetic lenses in the SEM column. Scanning coils near the end of the column direct and position the focused beam onto the sample surface. The electron beam is scanned in a raster pattern over the surface for imaging. The beam can also be focused at a single point or scanned along a line for x-ray analysis. The beam can be focused to a final probe diameter as small as about $10 \AA \AA$ [19]. To create an SEM image, the incident electron beam is scanned in a raster pattern across the sample's surface. The emitted electrons are detected for each position in the scanned area by an electron detector. The intensity of the emitted electron signal is displayed as brightness on a display monitor and/or in a digital image file. By synchronizing the position in the image scan to that of the scan of the incident electron beam, the display represents the morphology of the sample surface area. To examine the surface morphology of the nanoparticles samples were analyzed by SEM (JSM-6510LV, MA, USA). SEM analysis was performed at $1 \mathrm{kV}$ with magnifications ranging from $4000 \times$ to $12000 \times$. SEM images were used to analyze the interface between the drug and the polymer [20,21]. 


\section{Particle size distribution study}

Particle size can be determined by measuring the random changes in the intensity of light scattered from a suspension or solution. This technique is commonly known as dynamic light scattering (DLS) but is also called photon correlation spectroscopy (PCS) and quasi-elastic light scattering (QELS). The latter terms are more common in older literature. DLS is most commonly used to analyze nanoparticles [22, 23]. Dynamic light scattering (DLS) is based on the Brownian motion of dispersed particles. When particles are dispersed in a liquid they move randomly in all directions. The principle of Brownian motion is that particles are constantly colliding with solvent molecules. These collisions cause a certain amount of energy to be transferred, which induces particle movement. Laser diffraction particle size analyzer (Zetasizer Nano S90, Malvern, UK) was used for the determination of the particle size as well as the particle size distribution of the nanoparticles. The Zetasizer uses the dynamic light scattering (DLS) principle to determine the particle size, and measurements were made using a detection angle of $90^{\circ}[24]$.

\section{Fourier transform infrared spectroscopy (FTIR)}

Fourier Transform Infrared Spectroscopy spectra of HES, HP-b-CD and their prepared nanoparticles were taken in the range of 4000 to $500 \mathrm{~cm}^{-1}$ (Mid-infrared region) on a Perkin Elmer device, equipped with ATR device (Shimadzu 8400S FTIR spectrometer, Japan) using Attenuated Total Reflectance (ATR) method. Smoothing of the spectra and baseline correction was applied [25].

\section{Differential scanning calorimetry (DSC)}

DSC determinations were carried out in a stream of nitrogen atmosphere on a DSC 60A differential scanning calorimeter (Shimadzu, Japan). The accurately weighed sample $(10 \mathrm{mg})$ was heated in hard-pressed plus perforated crucibles of aluminum, at a scanning rate of $10^{\circ} \mathrm{C} \mathrm{min}^{-1}$ and the $50 \mathrm{ml} \mathrm{min}^{-1}$ flow rate of nitrogen. Consistent with standard procedures, the machine was calibrated for temperature as well as sensitivity with indium $[26,27]$.

\section{In vitro dissolution studies}

Dissolution testing is a requirement for all solid oral dosage forms and is used in all phases of development for product release and stability testing. It is a key analytical test used for detecting physical changes in an active pharmaceutical ingredient (API) and the formulated product. At the early stages of development, in vitro dissolution testing guides the optimization of drug release from formulations [28-31]. The dissolution studies for rilpivirine and its prepared nanoparticles were performed using a USP paddle apparatus. To mimic the conditions from the human gastrointestinal tract, the dissolution tests were carried out in a simulated medium [0.5\% Polysorbate 20 in $0.01 \mathrm{~N} \mathrm{HCl}(\mathrm{pH}=1.2)]$. The agitating speed was set at $100 \mathrm{rpm}$ and the temperature was kept at $37 \pm 0.5{ }^{\circ} \mathrm{C}$. Samples were withdrawn at a time interval of every $1 \mathrm{hr}$ up to $24 \mathrm{~h}$. Aliquots of $5 \mathrm{ml}$ were withdrawn from the apparatus and the same quantity of fresh dissolution medium was replaced at each sampling time. The solutions were immediately filtered through a $0.45 \mathrm{~mm}$ membrane filter, suitably diluted and the concentration of rilpivirine was measured by UV spectrophotometer at $304 \mathrm{~nm}$ against a blank containing the fresh dissolution medium. Suitable constructed standard curves were used. All dissolution measurements were performed in triplicate. The dissolution study plots were constructed by plotting the mean values of the cumulative dissolution of rilpivirine vs. time.

\section{RESULTS}

\section{Solubility study}

The solubility of rilpivirine in deionized water and dissolution medium. The solubility of rilpivirine in water and dissolution medium at $37{ }^{\circ} \mathrm{C} \pm 1{ }^{\circ} \mathrm{C}$ was found to be $3.93 \mathrm{mg} / \mathrm{ml}$ and 3.63 $\mathrm{mg} / \mathrm{ml}$ respectively. The solubility data of drugs in the dissolution medium confirm the presence of sink conditions in the media.

\section{Preliminary investigation in the formulation of nanoparticles}

Results of preliminary batches prepared for nanoparticle formulation was indicated in table 3. Preliminary batches were prepared to understand the effects of chitosan and TPP over the particle size and entrapment efficiency when used together. The preliminary batches were analyzed and the amount of $0.25 \%$ TPP was finalized to be $10 \mathrm{ml}$ for further studies.

\section{Characterization of formulation}

\section{Particle size and PDI}

The nanoparticles prepared were analyzed for their size and relevant PDI was determined with the help of Malvern Zetasizer Nano ZS-90. The codes denote the ratio of drug to chitosan solution in the ascending order from 1:1 to 1:6. The particle sizes of the nanoparticles were from $130.30 \pm 5.29$ to $350.80 \pm 13.17 \mathrm{~nm}$. The results were shown in table 4.

Table 3: Optimization of development parameters

\begin{tabular}{lllll}
\hline Batch & Chitosan $\mathbf{0 . 1}$ \% w/v (ml) & $\mathbf{0 . 2 5}$ \% TPP solution (ml) & Particle size (nm) $^{*}$ & \% Entrapment efficiency $^{*}$ \\
\hline M-1 & 15 & 10 & $560 \pm 12.02$ & $70.12 \pm 4.02$ \\
M-2 & 15 & 08 & $720 \pm 14.45$ & $62.45 \pm 3.12$ \\
M-3 & 15 & 06 & $840 \pm 11.30$ & $59.32 \pm 5.32$ \\
M-4 & 20 & 10 & $785 \pm 10.85$ & $65.78 \pm 6.82$ \\
M-5 & 20 & 08 & $876 \pm 12.28$ & $62.45 \pm 2.98$ \\
M-6 & 20 & 06 & $942 \pm 15.40$ & $59.32 \pm 6.36$ \\
\hline
\end{tabular}

${ }^{*}$ Values of particle size and \% Entrapment efficiency was presented as mean $\pm \operatorname{SD}(n=3)$

Table 4: Particle size distribution data for rilpivirine loaded chitosan nanoparticles

\begin{tabular}{lll}
\hline Batch code & Particle size $(\mathbf{n m})^{*}$ & PDI $^{*}$ \\
\hline MJ-1 & $154.90 \pm 4.40$ & $0.82 \pm 0.06$ \\
MJ-2 & $130.30 \pm 5.29$ & $0.98 \pm 0.11$ \\
MJ-3 & $141.30 \pm 8.81$ & $0.73 \pm 0.06$ \\
MJ-4 & $207.60 \pm 6.60$ & $0.81 \pm 0.02$ \\
MJ-5 & $190.32 \pm 6.60$ & $0.77 \pm 0.12$ \\
MJ-6 & $350.80 \pm 13.17$ & $0.66 \pm 0.04$ \\
\hline
\end{tabular}

${ }^{*}$ Values of particle size and PDI were presented as mean \pm SD $(n=3)$.

\section{Zeta potential}

Zeta potential was determined by Zetasizer (Malvern Instruments, Malvern, UK) using a disposable potential cuvette. For the selected batch MJ-2 sample, the mean zeta potential was found to be $25.38 \pm 2.18 \mathrm{mV}$ and polydispersity of all formulation was closer to 1 .

The results of the zeta potential were presented in table 5 . 
Table 5: Zeta potential of rilpivirine loaded chitosan nanoparticles

\begin{tabular}{ll}
\hline Batch code & Zeta potential $(\mathbf{m V})^{*}$ \\
\hline MJ-1 & $12.80 \pm 1.06$ \\
MJ-2 & $25.38 \pm 2.18$ \\
MJ-3 & $16.20 \pm 3.22$ \\
MJ-4 & $18.70 \pm 2.68$ \\
MJ-5 & $17.10 \pm 3.68$ \\
MJ-6 & $20.52 \pm 3.74$ \\
\hline
\end{tabular}

${ }^{*}$ Values of zeta potential was presented as mean \pm SD $(n=3)$.

\section{Scanning electron microscopy (SEM)}

Morphological characteristics of rilpivirine loaded Chitosan nanoparticle were analyzed by Scanning electron microscopy (SEM). Fig. 1 displayed SEM images for rilpivirine loaded chitosan nanoparticles.

\section{Entrapment efficiency}

Entrapment efficiency was calculated to determine the amount of rilpivirine entrapped inside the nanoparticle or been adsorbed on its surface. $10 \mathrm{mg}$ of nanoparticles were suspended in $10 \mathrm{ml}$ polysorbate-20 in $0.01 \mathrm{~N} \mathrm{HCl}(\mathrm{pH} \mathrm{1.2)}$ to extract the drug from nanoparticles. The mixture was stirred vigorously and been kept overnight at room temperature. The solution was then centrifuged and the supernatant was collected carefully using a micropipette. Solutions were then analyzed by a UV-Vis spectrophotometer at 304 $\mathrm{nm}$ with suitable dilutions. The data of drug entrapment efficiency of drug-loaded nanoparticles were as shown in table 6 the $\%$ encapsulation efficiencies of the nanoparticles were found in the range from $65.85 \pm 0.45$ to $78.40 \pm 0.10$.

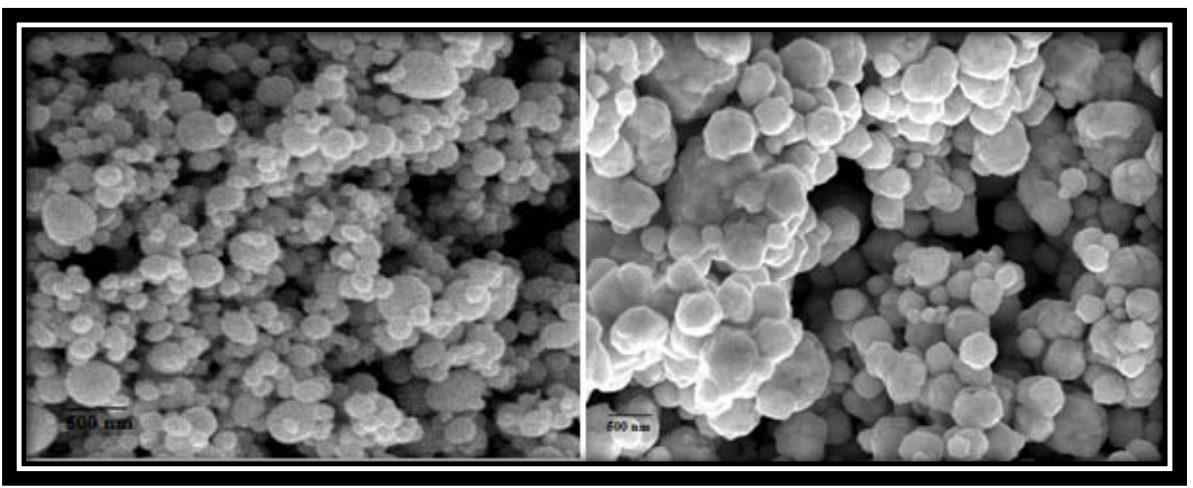

Fig. 1: SEM photographs for rilpivirine loaded chitosan nanoparticles MJ-2

Table 6: Entrapment efficiency of rilpivirine loaded chitosan nanoparticles

\begin{tabular}{ll}
\hline Batch code & EE \%* \\
\hline MJ-1 & $76.99 \pm 0.05$ \\
MJ-2 & $77.10 \pm 0.50$ \\
MJ-3 & $78.40 \pm 0.10$ \\
MJ-4 & $77.23 \pm 0.84$ \\
MJ-5 & $66.43 \pm 0.66$ \\
MJ-6 & $65.85 \pm 0.45$ \\
\hline
\end{tabular}

${ }^{*}$ Values of \% Entrapment efficiency was presented as mean \pm SD $(n=3)$.

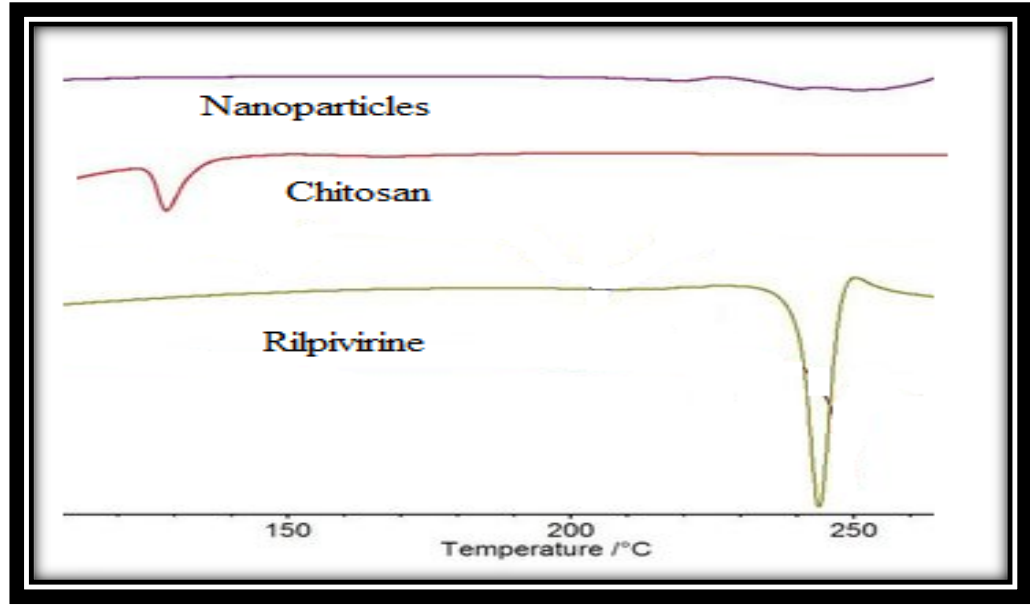

Fig. 2: DSC thermogram of rilpivirine, chitosan, and nanoparticles 


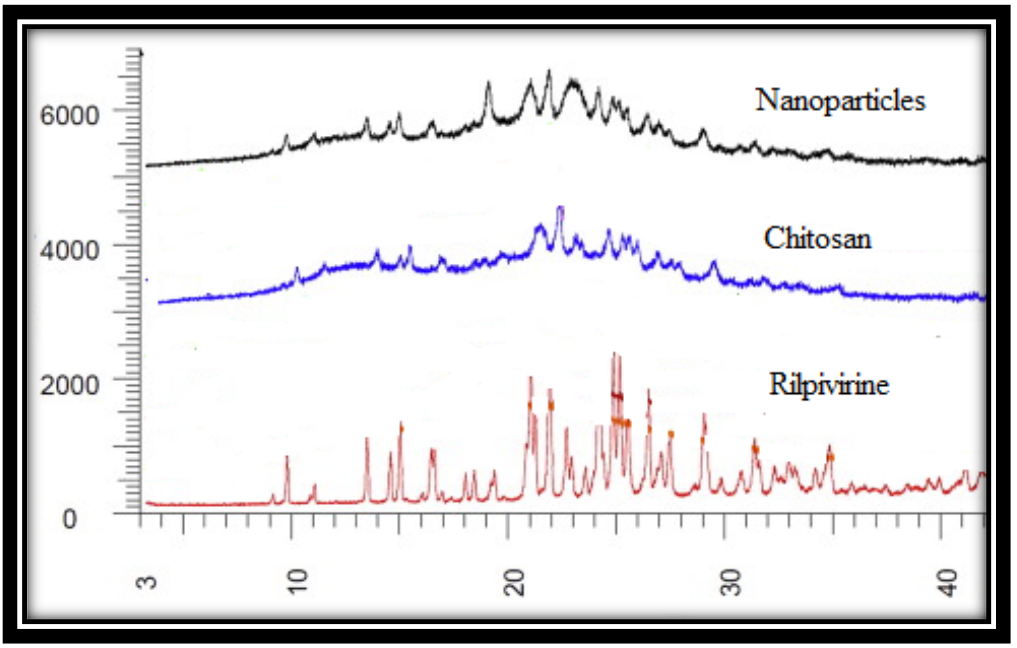

Fig. 3: FTIR spectra of rilpivirine, chitosan, and nanoparticles

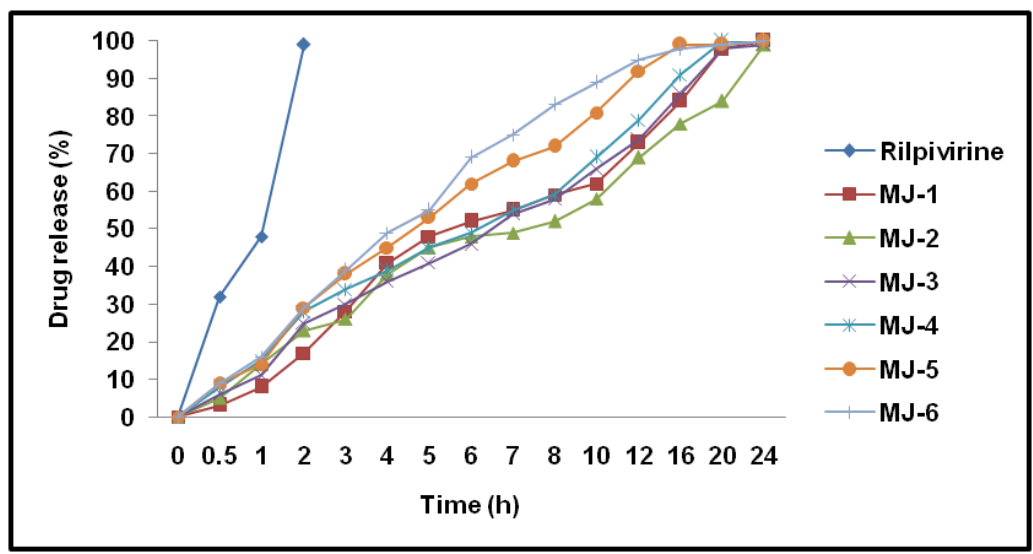

Fig. 4: Dissolution profile of rilpivirine and rilpivirine loaded chitosan nanoparticles

\section{DSC thermogram}

The DSC thermogram was utilized to study the changes over the structure of the drug and chitosan. The DSC thermogram of rilpivirine (fig. 2) showed an endothermic $\mathrm{T}_{\max }$ of $249^{\circ} \mathrm{C}$, corresponding to the melting point of the crystalline form of rilpivirine. Rilpivirine melts with decomposition which starts at about $249{ }^{\circ} \mathrm{C}$. The DSC thermogram of chitosan shows an endothermic $\mathrm{T}_{\max }$ of $78^{\circ} \mathrm{C}$.

\section{FTIR spectra}

Fig. 3 illustrated the FTIR spectra of rilpivirine, chitosan, and nanoparticles. The IR spectrum of rilpivirine is characterized by typical absorption bands at about $2217 \mathrm{~cm}^{-1}(\mathrm{C}-\mathrm{N}), 1652 \mathrm{~cm}^{-1}$ (C-O stretch) and $1497 \mathrm{~cm}^{-1}$ (aromatic C-C).

\section{In vitro drug release study}

The dissolution curves of rilpivirine and nanoparticles in $0.5 \%$ Polysorbate 20 in $0.01 \mathrm{~N} \mathrm{HCl}(\mathrm{pH}=1.2)$ at $37 \pm 0.5^{\circ} \mathrm{C}$ are shown in fig. 4. It was observed that more than $45 \%$ of the pure rilpivirine was released in $50 \mathrm{~min}$. After $90 \mathrm{~min}$ almost about $95 \%$ and after 120 min complete rilpivirine was released. While the in vitro release profile of nanoparticles was found to be sustained up to $24 \mathrm{~h}$.

\section{DISCUSSION}

Preliminary batches of the rilpivirine nanoparticles were prepared to finalize the concentration of TPP. It was observed that with the increase in the amount of $0.1 \%$ chitosan volume, irregular particle formation was observed. It also increased the particle size and decreased the entrapment efficiency, with every increase in the chitosan amount. Hence, $10 \mathrm{ml} 0.25 \%$ TPP with $15 \mathrm{ml}$ of $0.1 \%$ chitosan was finalized for the preparation of nanoparticles as it showed lower particle size and higher entrapment efficiency. The results of particle size distribution study indicated an irregular pattern in the particle size distribution, but a bigger picture of the increase in the particle size is observed with an increase in the chitosan amount $[1,3,5]$. Batch MJ-2 was found to be having the least particle size and based on that it was selected as the optimized batch. Nanoparticles formulation shows a narrow size distribution with a lower standard deviation. Zeta potential and polydispersity index showed that all particles in the formulation were in the acceptable range, inferring to be a stable formulation. The magnitude of the zeta potential is predictive of colloidal stability. Nanoparticles with zeta potential values greater than $+25 \mathrm{mV}$ typically have high degrees of stability [30, 31]. The SEM images (fig. 1) revealed that the nanoparticles were spherical and no agglomeration was observed indicating its stability. SEM images of obviously indicated the interface of drug with a polymeric material and accomplished the amalgamation of the drug in matrix material [11]. In the DSC thermograms of nanoparticles the sharp melting point peak of pure rilpivirine at $249^{\circ} \mathrm{C}$ and was not noticeable. The characteristic features of the rilpivirine peak were lost in the nanoparticles [14]. This phenomenon indicates that rilpivirine was molecularly detached and was no longer present there as a crystalline material, but was changed into the amorphous state. It also reveals that there was no free rilpivirine other than those entrapped in the nanoparticles confirming over its entrapment and stability [18]. In the results of FTIR spectra, there was a decrease of peak intensities is experiential in nanoparticles representative physically powerful physical interaction of the drug with carrier materials. However, no additional peaks were observed in any of the 
binary systems, indicating the absence of any chemical interface among rilpivirine and the chitosan [20]. The in vitro dissolution study for nanoparticles indicated that it sustains the drug release (fig. 4), for MJ-1 release up to $20 \mathrm{~h}$ was observed, whereas for MJ-2 it increased to $24 \mathrm{hr}$. For MJ-3 and MJ-4, the complete release was observed after $20 \mathrm{~h}$ and for MJ-5 and MJ-6, it was observed after 16 $h$. The data suggests that there is no direct relationship between increasing the chitosan solution in the nanoparticle with sustained release. As they show a decreased release profile with an increase in the ratio, there should be coordination between the particle size and the release profile. It was observed that with an increase in the particle size there was a decreased period in their successful complete release. The release pattern was found to be similar in all the batches of nanoparticles formulated [21].

\section{CONCLUSION}

In conclusion, the present study focused on the preparation of rilpivirine loaded nanoparticles containing Chitosan using the ionic gelation method for HIV infections. Nanoparticles were characterized by Fourier transform infrared spectroscopy (FTIR), differential scanning calorimetry (DSC), scanning electron microscopy (SEM) and in vitro drug release. The optimized nanoparticles were found with a particle size of $130.30 \pm 5.29 \mathrm{~nm}$. The entrapment efficiency (\% EE) of the chitosan nanoparticles were found to be $77.10 \pm 0.50 \%$. Morphological structures were studied using scanning electron microscopy which exposed the particles to be spherical with uniform size. It was observed that the nanoparticles formulated showed the absence of the crystalline nature of the drug and its switch to the amorphous state, which was confirmed through SEM and FTIR. Results showed that more than $45 \%$ of the pure drug was released in $50 \mathrm{~min}$ and after $90 \mathrm{~min}$ almost about $95 \%$ of the drug is released. While the in vitro release profile of chitosan nanoparticles was found to be sustained up to 24 hr. Sustained release of the rilpivirine could improve patient obedience to drug regimens, growing action effectiveness. Stability study revealed that the nanoparticles to be stable.

\section{FUNDING}

Nil

\section{AUTHORS CONTRIBUTIONS}

The authors of this manuscript share an equal contribution in all stages of the manuscript up to the approval of the final version.

\section{CONFLICT OF INTERESTS}

The authors of this manuscript do not have any financial or nofinancial conflicts of interest.

\section{REFERENCES}

1. Urnovitz HB, Murphy WH. Human endogenous retroviruses: nature, occurrence, and clinical implications in human disease. Clin Microbiol Rev 1996;1:72-99.

2. De Cock KM, Jaffe HW, Curran JW. The evolving epidemiology of HIV/AIDS. AIDS 2012;26:1205-13.

3. Connor RI, Paxton WA, Sheridan KE, Koup RA. Macrophages and CD4+T lymphocytes from two multiply exposed, uninfected individuals resist infection with primary nonsyncytium-inducing isolates of human immunodeficiency virus type 1. J Virol 1996;70:8758-64.

4. Liu R, Paxton WA, Choe S, Ceradini D, Martin SR. Homozygous defect in HIV 1 coreceptor accounts for resistance of some multiply exposed individuals to HIV-1 infection. Cell 1996;86:367-77.

5. Gupta RK, Van de Vijver DAMC, Manicklal S, Wainberg MA. Evolving uses of oral reverse transcriptase inhibitors in the HIV-1 epidemic: from treatment to prevention. Retrovirology 2013;10:82-5

6. Achhra AC, Boyd MA. Antiretroviral regimens sparing agents from the nucleoside (tide) reverse transcriptase inhibitor class: a review of the recent literature. AIDS Res Ther 2013;10:33.

7. O'Brien WA. Resistance against reverse transcriptase inhibitors. Clin Infect Dis 2000;30:185-92.
8. Paraschiv S, Otelea D, Baicus C, Tinischi M, Costache M. Nucleoside reverse transcriptase inhibitor resistance mutations in subtype F1 strains isolated from heavily treated adolescents in Romania. Int J Infect Dis 2009;13:81-9.

9. Wood E, Hogg RS, Lima VD, Kerr T, Yip B. Highly active antiretroviral therapy and survival in HIV infected injection drug users. JAMA 2008;300:550-4.

10. Puhan MA, Van Natta ML, Palella FJ, Addessi A, Meinert C. Excess mortality in patients with AIDS in the era of highly active antiretroviral therapy: temporal changes and risk factors. Clin Infect Dis 2010;51:947-56.

11. Sharma R, Jhorar R, Goyal K, Kumar R, Sharma AK Nanotechnological interventions in HIV drug delivery and therapeutics. Biointerface Res Appl Chem 2014;4:820-31.

12. Vedha Hari BN, Devendharan K, Narayanan N. Approaches of novel drug delivery systems for Anti-HIV agents. Int J Drug Dev Res 2013;5:16-24.

13. Mallipeddi R, Rohan LC. Progress in antiretroviral drug delivery using nanotechnology. Int J Nanomed 2010;5:533-47.

14. Das Neves J, Amiji MM, Bahia MF, Sarmento B. Nanotechnologybased systems for the treatment and prevention of HIV/AIDS. Adv Drug Delivery Rev 2010;62:458-77.

15. Mahajan SD, Aalinkeel R, Law WC, Reynolds JL, Nair BB, Sykes DE, et al. Anti-HIV-1 nanotherapeutics: promises and challenges for the future. Int J Nanomed 2012;7:5301-14.

16. Ojewole E, Mackraj I, Naidoo P, Govender T. Exploring the use of novel drug delivery systems for antiretroviral drugs. Eur J Pharm Biopharm 2008;70:697-710.

17. Vyas TK, Shah L, Amiji MM. Nanoparticulate drug carriers for delivery of HIV/AIDS therapy to viral reservoir sites. Expert Opin Drug Delivery 2006;3:613-28.

18. Shahiwala A, Amiji MM. Nanotechnology-based delivery systems in HIV/AIDS therapy. Future HIV Ther 2007;1:49-59.

19. Lanao JM, Briones E, Colino CI. Recent advances in delivery systems for anti-HIV1 therapy. J Drug Target 2007;15:21-36.

20. Govender T, Ojewole E, Naidoo P, Mackraj I. Polymeric nanoparticles for enhancing antiretroviral drug therapy. Drug Delivery 2008;15:493-501.

21. Iannazzo D, Pistone A, Galvagno S, Ferro S, De Luca L. Synthesis and anti-HIV activity of carboxylated and drug-conjugated multi-walled carbon nanotubes. Carbon 2015;82:548-61.

22. Vyas TK, Shah L, Amiji MM. Nanoparticulate drug carriers for delivery of HIV/AIDS therapy to viral reservoir sites. Expert Opin Drug Delivery 2006;3:613-28.

23. Kinch MS, Patridge E. An analysis of FDA-approved drugs for infectious disease: HIV/AIDS drugs. Drug Discovery Today 2014;19:1510-3.

24. Sharma M, Walmsley SL. Antiretroviral therapy in HIV/AIDS. Expert Opin Pharmacother 2014;15:395-405.

25. Piperno A, Giofre SV, Iannazzo D, Romeo R, Romeo G. Synthesis of C 4'Truncated phosphonated carbocyclic 2'-0xa$3^{4}$-azanucleosides as antiviral agents. J Org Chem 2010; 75:2798-805

26. Romeo R, Giofre SV, Macchi B, Balestrieri E, Mastino A. Truncated reverse isoxazolidinyl nucleosides: a new class of allosteric HIV-1 reverse transcriptase inhibitors. Chem Med Chem 2012;7:565-9.

27. Romeo R, Carnovale C, Giofre SV, Romeo G, Macchi B. Truncated phosphonated C-1'-branched N,O-nucleosides: a new class of antiviral agents. Bioorg Med Chem 2012;20:3652-7.

28. Romeo R, Carnovale C, Giofre SV, Monciino G, Chiacchio MA. Enantiomerically pure phosphonated carbocyclic 2'-oxa-3'azanucleosides: synth and bio eva. Molecules 2014;19:14406-16.

29. Mehellou Y, de Clercq E. Twenty-six years of anti-hiv drug discovery: Where do we stand and where do we go? J Med Chem 2010;53:521-38.

30. Thomas l, Mathew S. Biosynthesis, characterization, and antiinflammatory study of hemigraphis colorata loaded silver nanoparticles. Asian J Pharma Clin Res 2019;12:188-92.

31. Pavithra K, Bhagawati ST, Manjunath K. Development and evaluation of tizanidine hydrochloride loaded solid lipid nanoparticles. Asian J Pharma Clin Res 2019;12:152-8. 